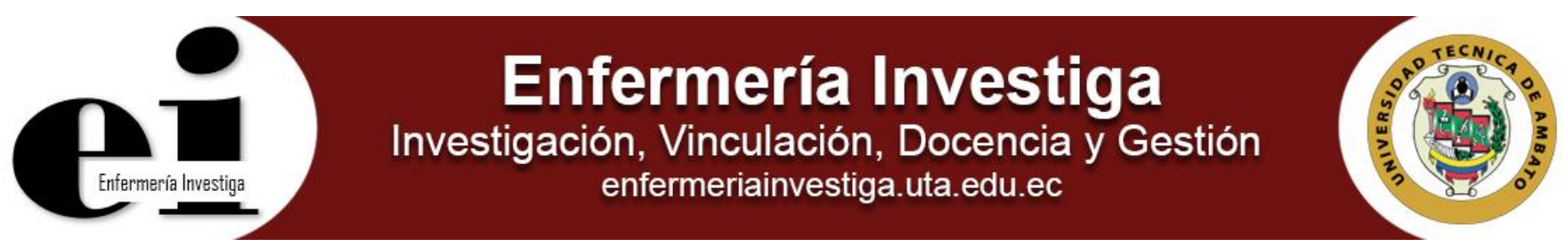

DOI: http://dx.doi.org/10.29033/ei.v2n4.2017.05

Artículo original

\title{
Percepción de riesgo en adolescentes relacionado con aborto
}

Perception of risk in adolescents related to abortion

\section{Bárbara Díaz Díaz¹, Yoslaidys Cordero Miranda¹, Romeo Gómez Aguado², Bertha Margarita Lorenzo Velázquez ${ }^{1}$}

${ }^{1}$ Universidad de Ciencias Médicas de Pinar del Río - Facultad de Ciencias Médicas - Carrera de Enfermería - Pinar del Río - Cuba

Díaz DB, Cordero MY, Gómez AR, Lorenzo VBM. Percepción de riesgo en adolescentes relacionado con aborto. Enferm Inv (Ambato). 2017 ; 2(4):145-149.

2477-9172 / 2550-6692 Derechos Reservados ( 2017 Universidad Técnica de Ambato, Carrera de Enfermería. Este es un artículo de acceso abierto distribuido bajo los términos de la Licencia Creative Commons, que permite uso ilimitado, distribución y reproducción en cualquier medio, siempre que la obra original es debidamente citada.

\section{Historia:}

Recibido: 06 noviembre 2017

Revisado: 30 noviembre 2017

Aceptado: 07 diciembre 2017

Palabras Claves: Adolescente;

sexualidad; aborto; riesgo

Keywords: Adolescent; sexuality; abortion; risk

\section{Resumen}

Introducción: Los estudios realizados sobre la educación de la sexualidad han permitido constatar que aún son insuficientes los conocimientos que poseen los adolescentes sobre aspectos relacionados con el tema, en especial los riesgos del aborto y su repercusión de manera desfavorable en la salud sexual.

Objetivo: Determinar la percepción de riesgo en adolescentes relacionado con aborto por embarazos no deseados.

Métodos: Estudio observacional descriptivo, transversal y prospectivo, se utilizaron encuestas y entrevistas, así como métodos estadísticos para el procesamiento de la información. El universo estuvo conformado por 354 adolescentes pertenecientes a los consultorios médicos 20, 28 y 30 del Grupo Básico de Trabajo 2 del policlínico Pedro Borrás Astorga, la muestra de 190 adolescentes se encontraron entre las edades de 14 a 18 años.

Resultados: Se evidenció una tendencia de los jóvenes a practicar conductas sexuales precoces y riesgosas, lo que favorece el aumento creciente de la práctica del aborto, con insuficientes conocimientos y percepción de riesgo sobre las consecuencias y riesgos de una interrupción de embarazo, donde se encuentran debilitados el rol de los padres y el papel del personal de salud de las áreas donde pertenecen.

Conclusiones: Se demostró que el proceso de educación para la salud muestra insuficiencias en cuanto a la creación de programas educativos más creativos que fomentan actividades de promoción y prevención de salud dirigidos a los adolescentes.

\begin{abstract}
Introduction: The studies carried out on the education of sexuality have made it possible to confirm that the knowledge that adolescents possess about aspects related to the subject is still insufficient, especially the risks of abortion and its adverse impact on sexual health.

Objetive: To determine the perception of risk in adolescents related to abortion due to unwanted pregnancies.

Methods: Descriptive, cross-sectional and prospective observational study, surveys and interviews were used, as well as statistical methods for the processing of information. The universe consisted of 354 adolescents belonging to the medical offices 20, 28 and 30 of the Basic Working Group 2 of the Pedro Borrás Astorga polyclinic, the sample of 190 adolescents were between the ages of 14 to 18 years.

Results: There was a tendency for young people to practice early and risky sexual behaviors, which favors the increasing increase in the practice of abortion, with insufficient knowledge and perception of risk on the consequences and risks of a pregnancy interruption, where they are weakened. role of parents and the role of health personnel in the areas where they belong.

Conclusions: It was shown that the health education process shows shortcomings in the creation of more creative educational programs that promote health promotion and prevention activities aimed at adolescents.
\end{abstract}

Autor de correspondencia:

Bertha Margarita Lorenzo Velázquez. Facultad de Ciencias Médicas, Universidad de Ciencias Médicas de Pinar del Río, Carretera Central Km 89 , Teléfono: +53 487 62481, Pinar del Río, Cuba. E-mail: bertham06@infomed.sld.cu 


\section{Introducción}

La investigación de la sexualidad no comienza hasta mediados del siglo pasado. Kinsey y los sucesores del instituto que lleva su nombre analizaron, mediante entrevistas, los diferentes comportamientos sexuales, en los que observaron las diferencias entre el comportamiento socialmente deseado y exigido, con el comportamiento real. Master y Jonson (1966) valoraron en el laboratorio y con diferente instrumental la fisiología y clínicas sexuales y diseñaron tratamientos novedosos para los problemas sexuales que actualmente siguen en vigor. Según señala Socarrás lbañez y otros, informes de entrevistas relevantes de aquella época fueron los de Hite (1976) donde se resumen las conductas y actitudes sexuales por grupos ${ }^{1,2}$.

Por otro lado, la sexualidad es una importante dimensión en la vida humana, fuente permanente en la gestión de comunicación, afecto y satisfacción, que influye de manera diferente en el modo y estado de salud de cada persona ${ }^{3}$. Esta toma especial connotación durante la adolescencia, pues el inicio cada vez más precoz de las relaciones sexuales, los hace vulnerables y susceptibles a embarazos no deseados, que terminan en interrupción, afectándose la salud sexual y reproductiva de este sector poblacional.

En muchas ocasiones, para combatir la fertilidad las mujeres acuden a la práctica del aborto, procedimiento que acompaña a la humanidad desde épocas inmemoriales. Este fenómeno social arroja estadísticas escalofriantes, sin embargo, si entráramos en el campo numérico veríamos que la cruda realidad que vive la sociedad actual es inmensamente mayor que las cifras que arrojan simples y vagos acercamientos ${ }^{2,3}$.

Aunque conceptualmente el aborto no es un método anticonceptivo, la realidad indica que su práctica se ha instalado entre las mujeres cubanas como un método que alterna con los demás métodos anticonceptivos e incluso, en mujeres muy jóvenes, antecede a la utilización de estos en no pocos casos. Ello explica por qué las tasas de aborto constituyen motivo de preocupación entre las autoridades sanitarias del país, y su uso excesivo es centro de reflexión y análisis profundo de numerosos especialistas ${ }^{4}$.

En términos de incidencia, la tasa estimada de abortos para América Latina y el Caribe, es de 65 abortos por cada 1000 mujeres en edad fértil, con cifras más altas en las áreas urbanas, tal incidencia implica una razón de por lo menos, un aborto de cada dos o tres partos, con tendencia al incremento y la Organización Mundial de la Salud (OMS) calcula que entre dos y cuatro millones de abortos en el mundo ocurren en adolescentes y el $13 \%$ de las muertes maternas en el mundo se deben a los abortos y sus complicaciones ${ }^{5}$.

En Cuba la tasa de abortos en menores de 20 años se ha mantenido históricamente por encima de la tasa global. Según la OMS, los abortos en la adolescencia constituyen un elemento fundamental que afecta la salud reproductiva de esta población, que si bien es cierto que desde su institucionalidad se han reducido las muertes maternas por esta causa, no se puede decir lo mismo de la morbilidad, pues secuelas a largo plazo como los embarazos ectópicos, los procesos inflamatorios pélvicos crónicos y la infertilidad; se han convertido en un lastre para estas mujeres y han afectado seriamente su salud reproductiva ${ }^{6,7}$.
Entre los participantes a quienes les concierne el aborto, es necesario considerar en primer lugar a las propias embarazadas, entre ellas las practicantes del aborto, el compañero sexual, los familiares, otras personas del contexto social y los prestadores de servicios de salud y educación, que pueden brindar luz sobre factores intrínsecos que es necesario considerar ${ }^{8}$.

Estos argumentos permiten revelar la existencia de contradicciones en el proceso de la educación de la sexualidad, expresadas en la falta de correspondencia entre los resultados actuales en la prevención del aborto en la adolescencia y las exigencias sociales contenidas en los objetivos generales del Programa Nacional de Atención Integral a la Salud del Adolescente. Esta situación obedece fundamentalmente a las carencias y falta de sistematicidad, que en el orden teórico y práctico tiene la concepción del proceso de la educación de la sexualidad, en función de incrementar el conocimiento y valoración de los riesgos del aborto; así como su utilización como método anticonceptivo ${ }^{9}$.

Este breve bosquejo sobre el tema invita al análisis y la reflexión de los programas de educación sexual vigentes, los cuales carecen de un enfoque biopsicosocial, de ahí la importancia de precisar las necesidades reales de los adolescentes y trabajar en base a elevar la percepción de riesgo, creando programas integrales que den solución a sus inquietudes y dudas, capaces de prepararlos para evitar problemas como el aborto producto de un embarazo a edad temprana, convirtiéndolos en jóvenes maduros y precavidos $^{8}$. El objetivo de este trabajo es determinar la percepción de riesgo en adolescentes relacionado con aborto por embarazos no deseados.

\section{Materiales y métodos}

El estudio se correspondió con un diseño observacional descriptivo, transversal y prospectivo. Se utilizaron métodos del nivel teórico como el histórico-lógico, análisis-síntesis, inducción-deducción y métodos empíricos como la revisión documental, encuestas y entrevistas como fuentes primarias de información, contando en todo momento con el consentimiento informado de los adolescentes y tutores. El universo estuvo conformado por 354 adolescentes pertenecientes a los consultorios médicos 20, 28 y 30 pertenecientes al Grupo Básico de Trabajo (GBT) 2 del policlínico Pedro Borrás Astorga, de la ciudad de Pinar del Río, Cuba. Se utilizó una muestra de 190 adolescentes que se encontraron entre las edades de 14 a 18 años, durante los años 2015 y 2016.

Como medida de resumen de la información se usaron las frecuencias absolutas y relativas porcentuales representadas en tablas.

\section{Resultados}

La tabla 1 muestra las vías por las cuales los adolescentes recibieron educación sexual. En orden decreciente la televisión, maestros y amigos, quedando los padres y el personal que labora en la atención primaria en un quinto y sexto lugar. 
Tabla 1. Vías por las que recibieron educación sexual. Policlínico Pedro Borrás Astorga, 2015-2016.

\begin{tabular}{lcc}
\hline $\begin{array}{l}\text { Fuente por la cual recibió la } \\
\text { educación sexual }\end{array}$ & $\mathbf{n}$ & $\%$ \\
\hline Televisión & 143 & 95.3 \\
Maestros & 127 & 84.7 \\
Amigos & 123 & 82.0 \\
Su pareja & 98 & 65.3 \\
Padres & 37 & 24.7 \\
Médico y enfermera familia & 29 & 19.3 \\
Otras vías & 17 & 11.3 \\
\hline
\end{tabular}

Fuente: Encuesta a adolescentes.

La tabla 2 muestra la edad en que los adolescentes mantuvieron la primera relación sexual. De un total de 190 encuestados el $46.8 \%$ de los adolescentes entre las edades de 16 a 18 años iniciaron sus primeras relaciones, seguidos de un $27.3 \%$ que hasta el momento de la encuesta no habían mantenido relaciones sexuales.

Tabla 2. Edad en la que los adolescentes mantuvieron la primera relación sexual.

\begin{tabular}{lcc}
\hline $\begin{array}{l}\text { Edad de la primera relación } \\
\text { sexual }\end{array}$ & $\mathbf{n}$ & $\%$ \\
\hline 14 a 15 & 49 & 25.7 \\
16 a 18 & 89 & 46.8 \\
No ha tenido relaciones sexuales & 52 & 27.3 \\
\hline Total & 190 & 100 \\
\hline Fuente: Encuesta a adolescentes.
\end{tabular}

En la tabla 3 se puede apreciar el criterio acerca del uso de algún método anticonceptivo durante las relaciones sexuales de los adolescentes, de un total de 190 encuestados, se presentan como resultados que solo el $33.1 \%$ se protegen durante el acto sexual y el $43.1 \%$ lo hacen de manera ocasional, hecho que se aproxima a lo reportado en otros estudios donde se evidencia la deficiente percepción de riesgo que poseen.

Tabla 3. Uso de anticonceptivos en las relaciones sexuales según sexo.

\begin{tabular}{lcccccc}
\hline Sexo & $\begin{array}{c}\text { Nunca } \\
(\mathbf{n})\end{array}$ & $\%$ & $\begin{array}{c}\text { Siempre } \\
(\mathbf{n})\end{array}$ & $\%$ & $\begin{array}{c}\text { Ocasionalmente } \\
(\mathbf{n})\end{array}$ & $\%$ \\
\hline $\mathrm{F}$ & 17 & 37.7 & 29 & 46.0 & 58 & 70.7 \\
M & 28 & 62.2 & 34 & 53.9 & 24 & 29.2 \\
\hline Total & $\mathbf{4 5}$ & $\mathbf{2 3 . 6}$ & 63 & 33.1 & 82 & 43.1 \\
\hline
\end{tabular}

cuesta a adolescentes.

F: Femenino. M: Masculino.

Al analizar el comportamiento de los adolescentes ante un embarazo se puede apreciar en la tabla 4 que el $56.8 \%$ de los encuestados consideró como principal vía de salida la interrupción del mismo, seguido por comunicar a los padres con un $34.7 \%$ (tabla 4).

Tabla 4. Comportamiento de los adolescentes ante un embarazo.

\begin{tabular}{lcc}
\hline $\begin{array}{l}\text { Comportamientos de los } \\
\text { adolescentes }\end{array}$ & $\mathbf{n}$ & $\%$ \\
\hline Comunicar a los padres & 66 & 34.7 \\
Abandonar los estudios & 9 & 4.7 \\
Continuar el embarazo & 17 & 8.9 \\
Interrumpir embarazo & 108 & 56.8 \\
Abandonar a la pareja & 51 & 26.8 \\
\hline Fuente: Encuesta a adolescentes
\end{tabular}

La tabla 5 muestra el número de abortos realizados en los adolescentes que ya mantienen relaciones sexuales, correspondiendo el mayor por ciento (39.4 y 25.2) a la práctica del mismo en una ocasión o más.

Tabla 5. Adolescentes según número de abortos realizados.

\begin{tabular}{lcc}
\hline Abortos & $\mathbf{n}$ & $\%$ \\
\hline Ninguno & 67 & 35.2 \\
Una vez & 75 & 39.4 \\
Dos veces & 48 & 25.2 \\
\hline Total & $\mathbf{1 9 0}$ & $\mathbf{1 0 0}$ \\
\hline Fuente: Encuesta a adolescentes. & &
\end{tabular}

\section{Discusión}

Los abortos en adolescentes, según el anuario estadístico del 2016 se han incrementado en Cuba, donde se registró una tasa de fecundidad en menores de 20 años, del 50 por cada mil mujeres ${ }^{10}$. En estudios similares se constató que las adolescentes cubanas abusan del aborto, la cifra se corresponde con más del $15 \%$ de la fecundidad total del país ${ }^{11}$.

En el presente estudio, la adolescencia intermedia concebida entre 15 y 17 años fue la más representativa del total de encuestados, siendo las adolescentes de esta edad las más vulnerables a la realización de abortos, sin dejar de considerar las significativas diferencias en su desarrollo biológico, psicológico y comportamiento social.

Paradójicamente la mayoría de los adolescentes encuestados refieren haber recibido información u orientación sexual, lo que se corresponde con estudios realizados en Cuba como el de los doctores Magaña y Quintero, que evidencian resultados similares, en los cuales un elevado por ciento de adolescentes poseen información sobre aspectos de la sexualidad, pero la práctica demuestra que el comportamiento sexual no se corresponde con estos, exponiéndose a riesgos cada vez a etapas más tempranas de la vida ${ }^{4,12}$

Los estudios de los doctores Piñeiro y Gómez, establecen que la mayoría de los adolescentes han recibido información a través de los padres, amigos, medios de difusión masiva y la escuela, considerando esta, aún insuficientes ${ }^{13,14}$. Al respecto, la política educativa que se está llevando en Cuba con los medios de divulgación masiva, fundamentalmente la televisión, constituye uno de los principales medios por donde los adolescentes reciben información sobre el tema.

Los padres juegan un papel importante en la comunicación con los hijos sobre todo en la etapa de la adolescencia. Se debe educar primero, pero siempre tratando de ganar confianza y que no se convierta en un problema, algo que es frecuente en la actualidad. Las relaciones sexuales aparecen más tempranamente, debiendo hablarse con los adolescentes sobre todo lo que concierne a estas, la importancia de protegerse no solo para evitar un embarazo, que finalmente puede terminar en un aborto; sino también para evitar las enfermedades de transmisión sexual que hoy día son la causa fundamental del cáncer cervicouterino ${ }^{3}$.

Los estudios más relevantes en la temática de educación de la sexualidad en función de prevenir el embarazo y por ende los abortos en la adolescencia, se han realizado desde el ámbito escolar, mientras que desde el Ministerio de Salud 
Pública (MINSAP) se encaminan al problema que representa para la salud, pero no se ha profundizado lo suficiente en el aspecto educativo; de manera que las estrategias y acciones no han surtido el efecto deseado. Así lo demuestran las estadísticas, que evidencian la necesidad e importancia del desarrollo de nuevas investigaciones en la temática ${ }^{11}$.

En la investigación desarrollada se palpó que cuando se analizan las edades en que los adolescentes comienzan las relaciones sexuales, los resultados son motivos de preocupación por el inicio precoz de esta práctica. Resultados que coinciden con un estudio realizado en el área de salud de Párraga, del municipio habanero de Arroyo Naranjo, donde existió una alta incidencia de embarazos, abortos y regulaciones menstruales debido a la iniciación temprana de las relaciones sexuales ${ }^{15}$.

Piñeiro refleja como negativo en su estudio que, la minoría de los adolescentes encuestados son de la opinión de que la primera relación sexual debe ser después de los 20 años, demostrándose la baja percepción de riesgo y un 30\% de los encuestados refirieron haber iniciado sus relaciones sexuales antes de los 15 años. Por su parte Quintero plantea que de un $20-25 \%$ de los adolescentes estudiados iniciaron sus relaciones sexuales en igual periodo ${ }^{12,13}$.

Los estudios sobre anticoncepción evidencian los niveles más bajos de práctica anticonceptiva entre los adolescentes en comparación con otras edades ${ }^{7}$. Es por ello que el adolescente promedio, con una frecuencia cada vez mayor, se expone al coito sin protección durante un año o más antes de solicitar anticoncepción y muchos esperan hasta que aparece un embarazo, para entonces solicitar interrupción del mismo como solución al problema.

En general, la percepción de riegos asociados a la práctica de relaciones sexuales sigue siendo baja. Llama la atención el hecho que aun cuando la protección durante las relaciones sexuales es escasa, el mayor porcentaje de los adolescentes reconocen el preservativo o condón como el método de más fácil acceso en su elección. Ello es consecuente con la publicidad que difunden los medios de comunicación masiva donde se reitera el eslogan difundido por el MINSAP: "Sin condón... ¡Ni pensarlo!"; mensaje orientado a la prevención de las infecciones de transmisión sexual (ITS), más que a prevenir los embarazos no planificados o no deseados ${ }^{9}$.

A pesar de las políticas del estado y del MINSAP por mejorar la calidad de vida de los adolescentes y de los programas de promoción y prevención de salud, debe continuarse con programas educativos dirigidos a los adolescentes, puesto que los mismos son más propensos a correr riesgos sexuales sin tomar en consideración sus posibles secuelas a largo plazo, habitualmente no planifican sus relaciones sexuales y estas ocurren en situaciones y lugares poco propicios, lo que conspira contra el uso de medidas profilácticas para evitar los embarazos no deseados y por consecuente los abortos ${ }^{16}$

\section{Conclusiones}

Se determinó que la percepción de riesgo en adolescentes relacionado con el aborto es baja, evidenciado con el inicio precoz de relaciones sexuales desprotegidas y uso excesivo de prácticas abortivas, lo que se traduce en una educación para la salud con insuficiencias en cuanto a la creación de programas educativos más creativos que fomentan actividades de promoción y prevención de salud dirigidos a los adolescentes.

\section{Conflicto de intereses}

Ninguno declarado por los autores.

\section{Agradecimientos}

Ninguno declarado por los autores.

\section{Referencias}

1. Master H, W, Johnson, $\mathrm{R}$ (1987). La reproducción humana. En sexualidad humana. Editorial Científico Técnica; Ministerio de cultura. La Habana.

2. Castro López FW. Atención de enfermería en el recién nacido. En Socarrás lbáñez $N$, Pérez Medina $M$, Leonard Castillo A, Alfonso Arenas N, Suárez Fuentes RR, Lemus Bocalandro O. Enfermería Ginecobstétrica. La Habana: Editorial Ciencias Médicas; 2009. p.260-4.

3. Bull Silva M, Della Martínez I, Periche Zaldivar A. Factores comunicativos desencadenantes del aborto en la adolescencia. Banes. Enero- julio 2011. CCCSS [en línea]. 2012 [citado 21 de diciembre de 2015]. Disponible en: http://www.eumed.net/rev/cccss/20/smz.html

4. Magaña Ml. Preocupación por el aumento de abortos y embarazos adolescentes en Cuba [en línea]. 2016 [citado 21 de diciembre de 2015]. Disponible en: http://www.actuall.com/vida/preocupacion-por-elaumento-de-abortos-y-embarazos-adolescentes-en-cuba/

5. Águila Setién S, Breto García A, Cabezas Cruz E, Delgado Calzado JJ, Santisteban Alba S. Obstetricia y Perinatología. Diagnóstico y tratamiento. La Habana: Editorial Ciencias Médicas; 2013.p. 4-10.

6. Álvarez Vázquez L, Salomón Avich N. El aborto en adolescentes en un contexto legal. Rev Cubana Salud Pública [en línea]. 2012 Mar [citado 26 de abril de 2016]. Disponible en http://scielo.sld.cu/scielo.php?script=sci_arttext\&pid=S0864$34662012000100005 \& \operatorname{lng}=\mathrm{es}$

7. Organización Mundial de la Salud. Salud de la madre, el recién nacido, del niño y del adolescente. Objetivo de Desarrollo del Milenio 5: Mejorar la salud materna [en línea]. 2015 [citado 21 de diciembre de 2015]. Disponible http://www.who.int/maternal child adolescent/topics/maternal/mdg/es/

8. Kahn JA, Huang B, Austin SB, Aweh GN, Colditz GA, Frazier AL. Development of a scale to measure adolescents beliefs and attitudes about postponing sexual initiation. J Adolescent Health [en línea]. 2012 [citado 26 de abril de 2016]. Disponible en: http://www.ncbi.nlm.nih.gov/pubmed/15488441

9. Benítez Pérez ME. La trayectoria del aborto seguro en Cuba: evitar mejor que abortar. Rev Nov Pob [en línea]. 2014; [citado 26 de abril de 2016]. Disponible http://scielo.sld.cu/scielo.php?script=sci_arttext\&pid=S181740782014000200007

10. Cuba. Anuario Estadístico de Salud 2016.Tasa de Fecundidad en mujeres menores de 20 años. La Habana; 2017.

11. González I. Adolescentes cubanas abusan del aborto [en línea]. 2016 [citado 2015 dic 21]. Disponible en: http://www.ipsnoticias.net/2013/09/adolescentes-cubanas-abusan-delaborto/

12. Quintero Paredes PP, Castillo Rocubert N, Roba Lazo BD, Padrón Gonzáles O, Hernandez Hierrezuelo ME. Estrategia de intervención educativa para prevenir el embarazo en la adolescencia. Revista de Ciencias Médicas de Pinar del Río [en línea]. 2012 [citado 16 de marzo de 2016]. Disponible en http://www.revcmpinar.sld.cu/index.php/publicaciones/article/view/877

13. Piñeiro Hernández $Y$. Caracterización del embarazo en la adolescencia Policlínico Pedro Borras 2014-2015. Trabajo de terminación de residencia para optar por el título de especialista de 1er grado en MGI.

14. Gómez Suárez RT, Rodríguez Hernández LM, Gómez Sarduy A, Torres Pestana E. Acciones educativas dirigidas a mejorar la percepción de riesgo del embarazo en adolescentes Revista Cubana de Salud Pública. [en línea] 2017 [citado 16 de marzo de 2016]. Disponible en: http://scielo.sld.cu/scielo.php?script=sci_arttext\&pid=S086434662017000200005

15. Enríquez Domínguez B, Puente Rizo E, Bustamante Diego $R$ Comportamiento de la regulación menstrual en la adolescente. Rev Cubana Med Gen Integr [en línea] 2012 [citado 16 de marzo de 2016]. Disponible 
Díaz DB/et al/Enfermería Investiga, Investigación, Vinculación, Docencia y Gestión-Vol. 2 No 42017 (Oct-Dic)

http://scielo.sld.cu/scielo.php?script=sci_arttext\&pid=S0864-

21252012000400013

16. Blanco Pereira ME, Jordán Padrón M, Pachón González L, Sánchez Hernández TB, Medina Robainas RE. Educación para la salud integral del adolescente a través de promotores pares. Rev Méd Electrón [en línea] 2011 [citado 16 de marzo de 2016]. Disponible en: http://www.revmatanzas.sld.cu/revista\%20medica/ano\%202011/vol3\%2 02011/tema12.htm

Enferm Inv. (Ambato) 2(4):145-149 